changes in AVP were correlated with PRA and PA. In only 2 of 24 patients, only AVP was markedly increased, just as seen in patients with SIADH. Therefore, AVP also seems to play only a permissive role in the development of edema in patients with liver cirrhosis.

\section{SUMMARY}

In the development of ascites and edema in liver cirrhosis, changes in renal hemodynamics and in renin secretion induced by hypoalbuminemia and portal hypertension seem to play an important role. However, aldosterone and antidiuretic hormone $(\mathrm{ADH})$ which have been believed to play an important role in the development of edema, are not necessarily increased in all patients with liver cirrhosis. Therefore, there is a possibility that aldosterone and ADH play only a permissive role in the development of ascites and edema in liver cirrhosis.

\title{
II. Clinic of Edema (4) Studies of Four Japanese Families with Hereditary Angioneurotic Edema (HANE) with Special Reference to the Mechanisms Involved in the Formation of Angioneurotic Edema
}

\author{
Junzo Kodama, MD and Shuji Funahashi, MD
}

Osaka National Hospital

In vitro studies of Cl-inhibitor (Cl-INH) have revealed its polyvalent biological activities, such as inhibition of Clr, Hageman factor, factor XI, plasmin and kallikrein. On the other hand, apart from excellent effectiveness of replacement therapy using Gl-INH-concentrate, the availability of testosteron, anticomplement-, antiplasminand antikallikrein-drugs in the treatment of HANE attacks causes a profound confusion in understanding mechanisms involved in the formation of edema in this hereditary disorder.

In an attempt to clarify this point, studies were undertaken on the changes of complement, fibrinolytic, clotting and kallikrein-kinin systems in comparison with the clinical course of HANE patients.

Biochemical parameters that reflect status of these systems were measured in 38 members (25 female and 13 male) of the four families. Furthermore, in proband of family I., determinations were done on occasions of her HANE attacks.

Members with plasma levels of Cl-INH protein above $10 \mathrm{mg} / \mathrm{dl}$ experienced no HANE attack. Between members with Cl-INH protein levels above $10 \mathrm{mg} / \mathrm{dl}$ and those below $10 \mathrm{mg} / \mathrm{dl}$, difference in $\mathrm{CH}_{60}$ titer, C2 hemolytic activity and Hageman factor activity was found statistically significant ( $p$ less than 0.01). Of 21 members with Cl-INH protein levels below $10 \mathrm{mg}$ / $\mathrm{dl}$, patients having experienced HANE attacks showed significantly lower Cl-INH protein levels, $\mathrm{CH}_{60}$ titer and $\mathrm{C} 2$ hemolytic

Jap J Med Vol 18, No 1 (Jan 1979) 


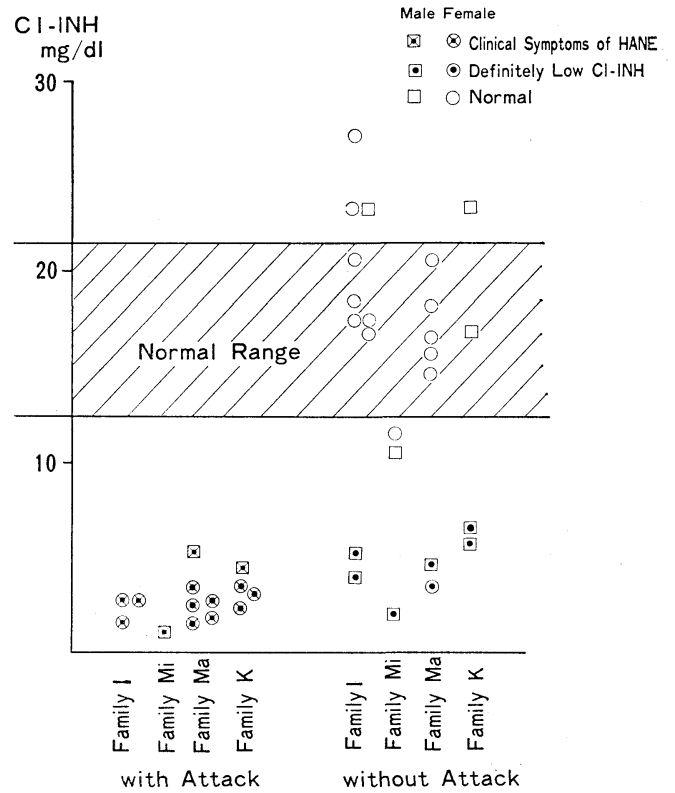

Fig. 1. Gl-1NH Protein in HANE Traits

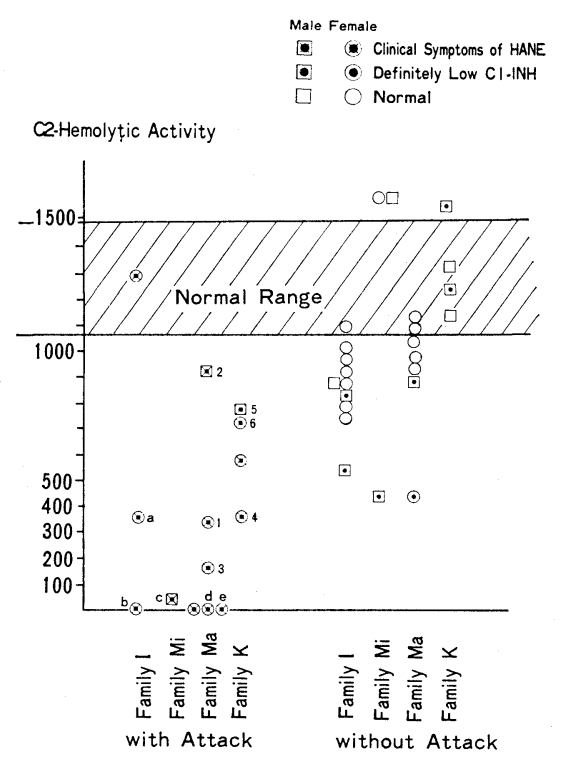

Fig. 2. C2-Hemolytic Activity in HANE-Traits activity than those having experienced no attack (p less than 0.01).

Then we tried to find out any difference in the readiness of suffering from HANE attack between female and male in these families. Fig. 1 shows that of 14 patients having experienced HANE attack with decreased Cl-INH protein, 10 are female and that of 7 members having experienced no HANE attack with decreased Cl-INH protein, 6 are male. Thus, females with decreased Cl-INH protein would be easier to have experienced HANE attack than males with the same trait. This sex difference in experiencing HANE attack is found statistically significant ( $p$ less than $0.01)$. This is of great interest, since the availability of testosteron and Danazol in the treatment of HANE has brought forward a problem in the mechanism of inheritance of this disease recently.

Fig. 2 shows the distribution of C2hemolytic activity in members of the families. Patients having experienced HANE attacks show generally low C2 hemolytic activity. Of these, members indicated by a, b, c, d and e have had serious attacks: two have had tracheotomy and one with an attack coming almost asphyxiated showed extremely low C2 hemolytic activity. Mild cases indicated by 1-6 and members having experienced no attack with decreased Cl-INH protein showed a slight decrease of C2 hemolytic activity. This tendency was found in the changes of C4 and Cl hemolytic activity as well. The vasoactive substance occurriing in reaction sequence of forming CII42 has been though as a mediator that increases vascular permeability in HANE patients. The decrease in C2 hemolytic activity together with the same changes in $\mathrm{Cl}$ and C4 hemolytic in patients experiencing serious attacks would be the result of enhanced catabolism of complement components. Another possibility for decreased hemolytic activities of C1, C4 and C2 would be impaired synthesis of proteins. This possibility would be discussed later together with changes in HMW (high molecular weight)-kininogen found in HANE families.

Fig. 3 shows the distribution of HMWkininogen levels in members of the four 


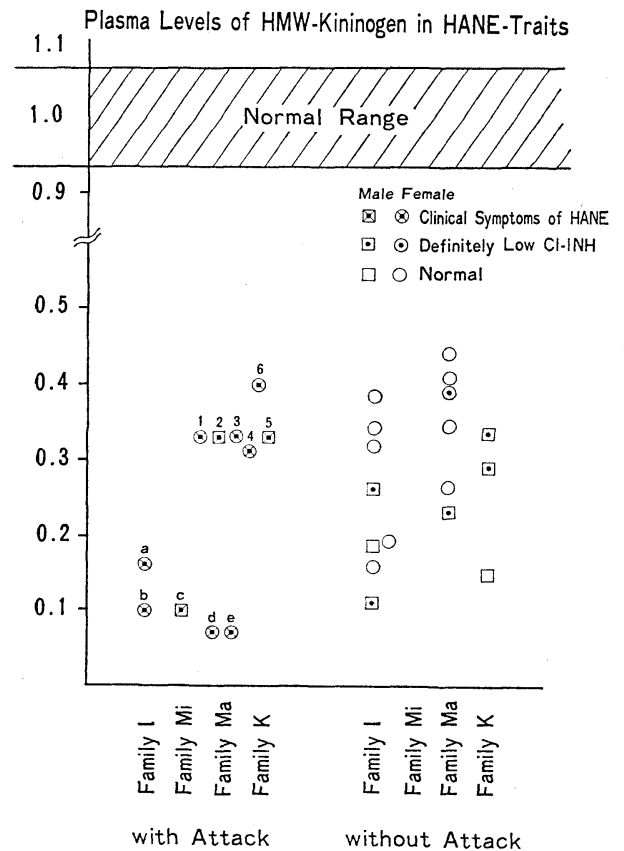

Fig. 3. Plasma Levels of HMW-

Kininogen in HANE-Traits

families. HMW-kininogen levels in all members tested showed significant decrease. In HANE patients, however, PTT was normal in remission and shortened on attack. This is the definite difference between HANE and Fitzgerald and/or Fujiwara traits. Teleologically, decrease in Cl, C4 and C.2 hemolytic activity as well as HMW-kininogen in HANE patients would benefit their pathological conditions forming edema and/or activating Hageman factor, since G1-INH lacks genetically. Since treatment of HANE patients with testosteron or Danazol was found to increase not only Cl-INH protein but C4, a possibility could be expected that this method of treatment would result in an increase of HMW-kininogen.

Taking mind on the correlation of HMW-kininogen levels to the HANE attack, a marked decrease of HMW-kinino- gen was found in cases indicated by $a, b$, c, $d$ and e. Members indicated by 1-6 and those having experienced no attack with the same trait showed normal levels of HMW-kininogon (normal means here that there is no difference between family members with normal Gl-INH protein levels in the families). Thus, in HANE patients having experienced severe attacks, bradykinin could take part in the formation of edema together with vasoactive substances originating from complement system.

In addition, proband of family I, who had experienced an attack of edema on her face following contusion, had showed an extremely low hemolytic activity of C2 before her attack (less than one unit). Nevertheless, she had experienced an attack of edema. Plasma levels of HMWkiningen decreased definitely following this attack of edema. Thus, possibilities that bradykinin could take part in formation of edema in HANE patient could not be ignored.

ACKNOWLEDGEMENT: To Professor Dr S Migita for his valuable suggestion, and to Professor emer. Dr T Yoshida and Professor Dr T Mizukawa for their kind encouragement in performing this work thaks the author sincerely.

\section{-COLLABORATORS-}

Center for Adult Diseases, Osaka Shinya Inai and Kazuyoshi Nagaki

Department of Pharmacology, School of Medicine, Kitasato University

Makoto Katori, Sachiko Oh-ishi and Yasuhiro Uchida

Second Department of Medicine, School of Medicine, Osaka City University

Masahisa Wada and Takami Ueno

Osaka National Hospital

Takenori Hashimoto, Keiichiro Yoshioka, yoko Matsumoto, Kikue Yasuno and Osamu Takamiya 